\title{
NORDHAUS-GADDUM-TYPE RESULTS FOR RESISTANCE DISTANCE-BASED GRAPH INVARIANTS
}

\author{
Kinkar CH. DAS \\ Department of Mathematics \\ Sungkyunkwan University \\ Suwon 440-746, Republic of Korea \\ e-mail: kinkardas2003@googlemail.com \\ YUJUN YANG ${ }^{1}$ \\ School of Mathematics and Information Science \\ Yantai University \\ Yantai, Shandong, 264005, PR China \\ e-mail: yangyj@yahoo.com \\ AND \\ KEXIANG Xu \\ College of Science \\ Nanjing University of Aeronautics \& Astronautics \\ Nanjing, Jiangsu, 210016, PR China \\ e-mail: kexxu1221@126.com
}

\begin{abstract}
Two decades ago, resistance distance was introduced to characterize "chemical distance" in (molecular) graphs. In this paper, we consider three resistance distance-based graph invariants, namely, the Kirchhoff index, the additive degree-Kirchhoff index, and the multiplicative degree-Kirchhoff index. Some Nordhaus-Gaddum-type results for these three molecular structure descriptors are obtained. In addition, a relation between these Kirchhoffian indices is established.
\end{abstract}

Keywords: resistance distance, Kirchhoff index, additive degree-Kirchhoff index, multiplicative degree-Kirchhoff index, Nordhaus-Gaddum-type result.

2010 Mathematics Subject Classification: 05C12, 05C90.

\footnotetext{
${ }^{1}$ Corresponding author.
} 


\section{REFERENCES}

[1] D. Bonchev, A.T. Balaban, X. Liu and D.J. Klein, Molecular cyclicity and centricity of polycyclic graphs. I. Cyclicity based on resistance distances or reciprocal distances, Int. J. Quantum Chem. 50 (1994) 1-20.

doi:10.1002/qua.560500102

[2] H. Chen and F. Zhang, Resistance distance and the normalized Laplacian spectrum, Discrete Appl. Math. 155 (2007) 654-661.

doi:10.1016/j.dam.2006.09.008

[3] H. Chen and F. Zhang, Resistance distance local rules, J. Math. Chem. 44 (2008) 405-417. doi:10.1007/s10910-007-9317-8

[4] P. Dankelmann, H.C. Swart and P. van den Berg, Diameter and inverse degree, Discrete Math. 308 (2008) 670-673.

doi:10.1016/j.disc.2007.07.053

[5] K.Ch. Das, I. Gutman and B. Zhou, New upper bounds on Zagreb indices, J. Math. Chem. 46 (2009) 514-521.

doi:10.1007/s10910-008-9475-3

[6] R.M. Foster, The average impedance of an electrical network, in: Contributions to Applied Mechanics (Edwards Bros., Michigan, Ann Arbor, 1949) 333-340.

[7] I. Gutman, L. Feng and G. Yu, Degree resistance distance of unicyclic graphs, Trans. Comb. 1 (2012) 27-40.

[8] I. Gutman and B. Mohar, The Quasi-Wiener and the Kirchhoff indices coincide, J. Chem. Inf. Comput. Sci. 36 (1996) 982-985.

doi:10.1021/ci960007t

[9] D.J. Klein, Graph geometry, graph metrics, \& Wiener, MATCH Commun. Math. Comput. Chem. 35 (1997) 7-27.

[10] D.J. Klein, Centrality measure in graphs, J. Math. Chem. 47 (2010) 1209-1223. doi:10.1007/s10910-009-9635-0

[11] D.J. Klein and O. Ivanciuc, Graph cyclicity, excess conductance, and resistance deficit, J. Math. Chem. 30 (2001) 271-287. doi:10.1023/A:1015119609980

[12] D.J. Klein and M. Randić, Resistance distance, J. Math. Chem. 12 (1993) 81-95. doi:10.1007/BF01164627

[13] D.J. Klein and H.-Y. Zhu, Distances and volumina for graphs, J. Math. Chem. 23 (1998) 179-195. doi:10.1023/A:1019108905697

[14] E.A. Nordhaus and J.W. Gaddum, On complementary graphs, Amer. Math. Monthly 63 (1956) 175-177. doi:10.2307/2306658 
[15] J.L. Palacios and J.M. Renom, Another look at the degree-Kirchhoff index, Int. J. Quantum Chem. 111 (2011) 3453-3455.

doi:10.1002/qua.22725

[16] H. Wiener, Structural determination of paraffin boiling points, J. Amer. Chem. Soc. 69 (1947) 17-20.

doi:10.1021/ja01193a005

[17] W. Xiao and I. Gutman, Resistance distance and Laplacian spectrum, Theor. Chem. Acc. 110 (2003) 284-289. doi:10.1007/s00214-003-0460-4

[18] Y. Yang, Relations between resistance distances of a graph and its complement or its contraction, Croat. Chem. Acta 87 (2014) 61-68.

doi: $10.5562 /$ cca2318

[19] Y. Yang, H. Zhang and D.J. Klein, New Nardhaus-Gaddum-type results for the Kirchhoff index, J. Math. Chem. 49 (2011) 1587-1598. doi:10.1007/s10910-011-9845-0

[20] B. Zhou and N. Trinajstić, A note on Kirchhoff index, Chem. Phys. Lett. 455 (2008) 120-123.

doi:10.1016/j.cplett.2008.02.060

[21] B. Zhou and N. Trinajstić, On resistance-distance and Kirchhoff index, J. Math. Chem. 46 (2009) 283-289.

doi:10.1007/s10910-008-9459-3

[22] H.-Y. Zhu, D.J. Klein and I. Lukovits, Extensions of the Wiener number, J. Chem. Inf. Comput. Sci. 36 (1996) 420-428.

doi:10.1021/ci950116s

Received 11 March 2015

Revised 31 October 2015

Accepted 31 October 2015 